\title{
Identification of Fruit Volatiles from Green Hawthorn (Crataegus Viridis) and Blueberry Hawthorn (Crataegus Brachyacantha) Host Plants Attractive to Different Phenotypes of Rhagoletis Pomonella Flies in the Southern United States
}

\author{
Dong H. Cha • Thomas H. Q. Powell • Jeffrey L. Feder • \\ Charles E. Linn Jr
}

Received: 2 March 2011 /Revised: 13 August 2011 /Accepted: 19 August 2011 /Published online: 2 September 2011

(C) Springer Science+Business Media, LLC 2011

\begin{abstract}
The apple maggot fly, Rhagoletis pomonella, infests several hawthorn species in the southern USA. In a companion paper, we showed that $R$. pomonella flies infesting two different mayhaw species (Crataegus opaca and $C$. aestivalis) can discriminate between volatile blends developed for each host fruit, and that these blends are different from previously constructed blends for northern fly populations that infest domestic apple (Malus domestica), downy hawthorn (Crataegus mollis), and flowering dogwood (Cornus florida). Here, we show by using coupled gas chromatography and electroantennographic detection (GCEAD), gas chromatography with mass spectrometry (GC$\mathrm{MS}$ ), and flight tunnel bioassays, that two additional southern hawthorn fly populations infesting $C$. viridis (green hawthorn) and C. brachyacantha (blueberry hawthorn) also can discriminate between volatile blends for each host fruit type. A 9-component blend was developed for $C$. viridis (3methylbutan-1-ol [5\%], butyl butanoate [19.5\%], propyl hexanoate [1.5\%], butyl hexanoate [24\%], hexyl butanoate [24\%], pentyl hexanoate [2.5\%], 1-octen-3-ol [0.5\%], pentyl butanoate [2.5\%], and (3E)-4,8-dimethyl-1,3,7-nonatriene (DMNT) [20.5\%]) and an 8-component blend for $C$. brachyacantha (3-methylbutan-1-ol [0.6\%], butyl acetate
\end{abstract}

D. H. Cha $\cdot$ C. E. Linn Jr $(\bowtie)$

Department of Entomology,

NYS Agricultural Experiment Station, Cornell University,

Geneva, NY 14456, USA

e-mail: CEL1@cornell.edu

T. H. Q. Powell • J. L. Feder

Department of Biological Sciences, University of Notre Dame,

Notre Dame, IN 46656, USA
[50\%], pentyl acetate [3.5\%], butyl butanoate [9\%], butyl hexanoate [16.8\%], hexyl butanoate [16.8\%], 1-octen-3-ol [0.3\%], and pentyl butanoate [3\%]). Crataegus viridis and $C$. brachyacantha-origin flies showed significantly higher levels of upwind oriented flight to their natal blend in flight tunnel assays compared to the alternate, non-natal blend and previously developed northern host plant blends. The presence of DMNT in $C$. viridis and butyl acetate in $C$. brachyacantha appeared to be largely responsible for driving the differential response. This sharp behavioral distinction underscores the diversity of odor response phenotypes in the southern USA, points to possible host race formation in these populations, and despite the presence of several apple volatiles in both blends, argues against a functional apple race existing on southern host plants prior to the introduction of apple to North America.

Key Words Apple maggot fly· Sympatric speciation · Host races · Southern hawthorns · Olfaction · Fruit odor discrimination $\cdot$ Flight tunnel

\section{Introduction}

The apple maggot, Rhagoletis pomonella Walsh (Diptera: Tephritidae) is a model system for understanding speciation without geographic isolation (i.e., sympatric speciation) via host plant shifting for phytophagous insects (Berlocher et al., 1993; Feder, 1998; Funk et al., 2002; Berlocher and Feder, 2002; Smadja and Butlin, 2009). In the northern USA, where the apple and hawthorn host races co-occur, downy hawthorn is the primary host for $R$. pomonella flies. 
However, the situation is different in the southern USA, where $R$. pomonella infests an array of several different hawthorn species that generally overlap in their geographic ranges, but differ in key traits such as fruiting time, host fruit color and size, and potentially fruit volatiles, thus presenting the opportunity for host-related divergence.

In a companion paper, we investigated the fruit volatile profiles for three related Crataegus species in the series Aestivales, collectively known under the common name of "mayhaw" in the South. Two of these species, the western mayhaw (C. opaca) and the eastern mayhaw (C. aestivalis), are infested by $R$. pomonella. A third described species, $C$. rufula, occurs primarily at the intersection of the eastern and western species' ranges between the lower Pearl River and Pascagoula River drainages in Mississippi and may represent a hybrid species; $C$. rufula does not appear to be infested by $R$. pomonella (Cha et al., 2011b, this issue). Our results are consistent with the possibility of endemic southern hawthorn-infesting host races of $R$. pomonella and do not support a "pre-adapted" apple race of the fly being present in the South (Cha et al., 2011b).

There are other hawthorn hosts of $R$. pomonella in the South, however, that could serve as a source for a preassembled apple race and/or shed additional insight into the evolutionary origins of apple volatile attraction. The fly also infests blueberry hawthorn, $C$. brachyacantha Englem. and Sarg. and green hawthorn, C. viridis L., as principal hosts. The blueberry hawthorn is so named for its characteristic dark blue, subglobose fruit. It is one of the tallest southern species in Crataegus, and is further distinguished by its small, waxy, crenulate-serrated leaves and small curved thorns (Sargent, 1933). Blueberry hawthorn fruit typically ripens from late August through September. Blueberry hawthorns are found in moist soil on the edge of forests bordering on low-lying prairies (Correll and Johnston, 1970). Its natural range is the "piney woods" region of eastern Texas through much of Louisiana, barely extending into adjacent counties in Arkansas and Mississippi.

The green hawthorn, $C$. viridis, has a broader range. It is found from southern Illinois southward to the coastal plain of Texas, eastward to the Florida panhandle and as far north as the Virginia piedmont. It is sparsely distributed through much of this range, only occurring in high densities west of the Mississippi River (Sargent, 1890). Green hawthorn is comprised of a complex of both sexual and apomictic individuals (Talent and Dickinson, 2005), and is distinguished from other hawthorns by dark green, acute, ovate leaves and small red-orange fruit (Vines, 1977). Green hawthorn has the latest fruiting phenology of southern hawthorns, with ripening not occurring until November. Unlike many hawthorn species, whose fruit abscises shortly after ripening, uninfested green hawthorn fruit often remains on the tree through winter. For this reason, a cultivar of green hawthorn known as "Winter King" has recently become popular with horticulturalists outside its native range.

Here, we expand on our previous studies and report on the identification of volatile blends for blueberry and green hawthorns through a combination of gas chromatographyelectroantennographic detection (GC-EAD) and gas chromatography-mass spectrometric (GC-MS) analysis of solid phase microextraction (SPME) and adsorbent volatile collection samples from whole fruit. Behavioral flight tunnel assays also were conducted using the blueberry and green hawthorn blends and our previously developed apple, downy hawthorn, and flowering dogwood blends to test for possible olfactory pre-adaptation of flies that infest these two southern hawthorn species to apple. We report results showing again that both blueberry and green hawthornorigin flies respond maximally to their natal fruit volatile blends and are relatively unresponsive to the alternative non-natal blends, consistent with endemic host race formation of $R$. pomonella on native southern hawthorns. The results also show that the volatile blend for green hawthorn flies, in particular, contains volatiles used by northern downy hawthorn and apple flies, and thus could be a potential source for genetic variation, thus contributing to olfactory fruit odor discrimination and host race formation in the North.

\section{Methods and Materials}

Details concerning methods and procedures for fruit volatile adsorbent and SPME sampling, GC-EAD and chemical analyses, synthetic blend development, and flight tunnel behavioral testing, can be found in the companion paper (Cha et al., 2011b, this issue). As done previously in Cha et al. (2011b), differences in the frequency of upwind flight in the tunnel were compared using Fisher's Exact Test in $\mathrm{R}$ ( $\mathrm{R}$ development core team, Vienna, Austria).

Insects Flies used in the study were collected as larvae in infested host fruit from three different blueberry and seven different green hawthorn sites in the South (Fig. 1; Table 1) and reared to adulthood in the Notre Dame laboratory (Neilson and McAllan, 1965; Dambroski and Feder, 2006). Upon eclosion, adult flies were kept in a walk-in environmental chamber on an artificial diet (Fein et al., 1982). Adult flies at 0-7 and 10-21-d-old were used for GC-EAD analyses and flight-tunnel behavior tests, respectively.

Fruit Blueberry hawthorn fruit was collected from Monroe, LA, USA, in 2007 (Fig. 1; Table 1). Green hawthorn fruit was collected from Dewey Wills Wildlife Management 


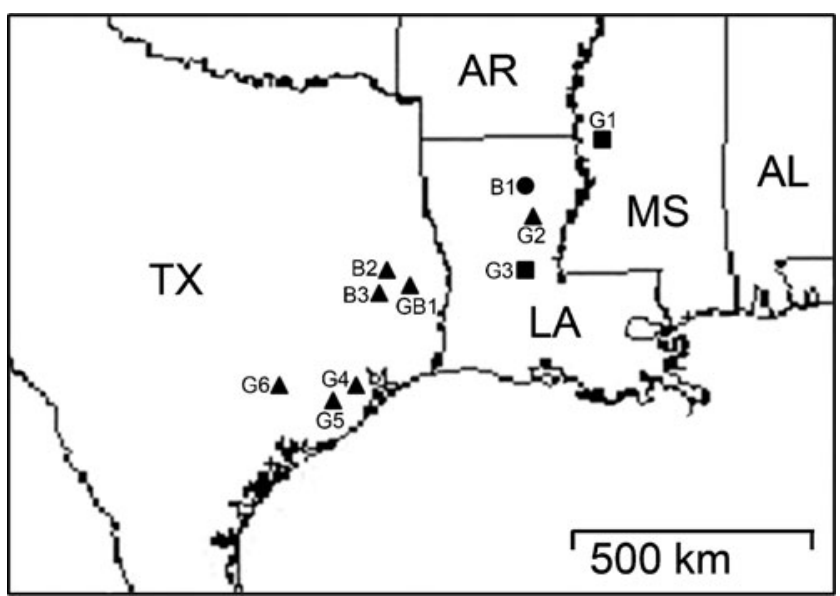

Fig. 1 Map of collection sites for blueberry hawthorn (B) and green hawthorn (G) fruit (circles), flies (triangles), or both (squares). Abbreviations for each of the southern States indicated are: AL, Alabama; AR, Arkansas; LA, Louisiana; MS, Mississippi; TX, Texas

Area, LA, in 2007 and Rolling Fork, MS, USA, in 2008 (Fig. 1; Table 1). Fruit collections were made off of trees in the field and shipped overnight to the Cornell lab for volatile characterization.

Chemicals Pentyl hexanoate, hexyl butanoate, butyl hexanoate, propyl hexanoate, pentyl butanoate, butyl butanoate, 1octen-3-ol, 3-methylbutan-1-ol, 3-methylbutyl acetate, dimethyl trisulfide, $\beta$-caryophyllene (purities $>98 \%$ ), and dihydro- $\beta$ ionone $(>90 \%)$ were purchased from Sigma-Aldrich (St. Louis, MO, USA); butyl acetate ( $>99 \%)$ and pentyl acetate $(>98 \%)$ from TCI America (Portland, OR, USA); ethyl acetate (99.9\%) from Fischer Scientific (Pittsburgh, PA, USA). The compound (3E)-4,8-dimethyl-1,3,7-nonatriene (DMNT) was synthesized by oxidation of geraniol and then by Wittig reaction with methylenetriphenylphosphorane (Greenwald et al., 1963) and purified (>97\% with $>97 \% E$-isomer by $\mathrm{GC} /$ MS) using flash chromatography on silica gel. The synthesis product was eluted with hexane and then subjected to Kugelrohr distillation.

Table 1 Collection sites for green hawthorn (Crataegus viridis) and blueberry hawthorn (Crataegus brachyacantha) fruit and flies as marked in Fig. 1

\begin{tabular}{lllll}
\hline Map Name & Host plant species & Location & Latitude & Longitude \\
\hline B1 & C. brachyacantha & Co. Rd 841, Ouachita Parish, LA & $32^{\circ} 19^{\prime} \mathrm{N}$ & $90^{\circ} 55^{\prime} \mathrm{W}$ \\
B2 & C. brachyacantha & SFA exp. forest, Nacogdoches Co, TX & $31^{\circ} 30^{\prime} \mathrm{N}$ & $94^{\circ} 46^{\prime} \mathrm{W}$ \\
B3 & C. brachyacantha & Lufkin, Angelina Co, TX & $31^{\circ} 20^{\prime} \mathrm{N}$ & $94^{\circ} 43^{\prime} \mathrm{W}$ \\
GB1 & Both & Marion Ferry Park, Angelina Co, TX & $31^{\circ} 30^{\prime} \mathrm{N}$ & $94^{\circ} 32^{\prime} \mathrm{W}$ \\
G1 & C. viridis & Hwy 16, Rolling Fork, Sharkey Co, MS & $32^{\circ} 54^{\prime} \mathrm{N}$ & $90^{\circ} 52^{\prime} \mathrm{W}$ \\
G2 & C. viridis & Fort Necessity, Caldwell Parish, LA & $32^{\circ} 02^{\prime} \mathrm{N}$ & $91^{\circ} 49^{\prime} \mathrm{W}$ \\
G3 & C. viridis & Dewey Wills WMA, La Salle Parish, LA & $30^{\circ} 49^{\prime} \mathrm{N}$ & $90^{\circ} 57^{\prime} \mathrm{W}$ \\
G4 & C. viridis & Brazos Bend SP, Fort Bend Co, TX & $32^{\circ} 28^{\prime} \mathrm{N}$ & $97^{\circ} 45^{\prime} \mathrm{W}$ \\
G5 & C. viridis & Cedar Lanes, Wharton Co, TX & $30^{\circ} 25^{\prime} \mathrm{N}$ & $95^{\circ} 21^{\prime} \mathrm{W}$ \\
G6 & C. viridis & Palmetto SP, Gonzalez Co, TX & $28^{\circ} 08^{\prime} \mathrm{N}$ & $96^{\circ} 59^{\prime} \mathrm{W}$ \\
\hline
\end{tabular}

Modified Synthetic Blends To determine the relative importance of individual volatiles in the blend, or those that might function as inter-specific behavioral antagonists for green and blueberry hawthorn-origin flies, we prepared six modified blends by adding and/or subtracting volatile compounds from the complete blends (Table 2). We concentrated on five different compounds: 3-methylbutan1-ol, butyl acetate, propyl hexanoate, DMNT, and 1-octen3-ol. Previous studies have shown that 3-methylbutan-1-ol is an essential volatile inducing agonistic behavior for northern downy hawthorn flies (Linn et al., 2005b) and also for southern mayhaw flies (Cha et al., 2011b, this issue). Butyl acetate was examined because it is present in the blueberry hawthorn, but not common in other hawthorn species found in blueberry hawthorn growing regions (e.g., western mayhaw, C. opaca). DMNT, 1-octen-3-ol, and propyl hexanoate are all compounds that are important for the northern apple, downy hawthorn, and dogwood host races. DMNT is unique to green hawthorn in the South and was previously shown to be essential for maximal upwind flight by northern downy hawthorn flies (Nojima et al., 2003a). The volatile 1-octen-3-ol is in both green and blueberry hawthorn, and is an essential volatile in the dogwood blend (Nojima et al., 2003b) but an antagonist to apple flies (Linn et al., 2005a). The modified blends were prepared so that each compound was always in the same amount as in corresponding complete blends, thus eliminating the potential effect of variation in concentration. For flight tunnel testing, we applied $200 \mu \mathrm{l}$ of the modified blend to a rubber septum. The concentration of each modified blend is listed in Table 2.

\section{Results}

Identification of Volatiles from Green and Blueberry Hawthorn Fruit Green and blueberry hawthorn fruit were analyzed by using SPME and adsorbent extracts with GC- 
Table 2 Relative ratio (\%) of volatile compounds in the complete green $(\mathrm{Gc})$ and blueberry $(\mathrm{Bc})$ synthetic blends and their modified blends (G1, G2, B1, B2, B3 and B4). We prepared the modified blends so that the same compound was always in the same amount as in corresponding complete blends, thus eliminating the potential effect of variation in concentration. For flight tunnel tests, $200 \mu \mathrm{l}$ of Gc or Bc (prepared at $1.0 \mu \mathrm{g} / \mu \mathrm{l}$ ), thus $200 \mu \mathrm{g}$ of total volatile compounds, were loaded to a rubber septum. Ratio and concentration of modified blends (G1, G2, B1, B2, B3 and B4) were formulated as found in Gc or Bc. For flight tunnel tests of the modified blends, we also loaded $200 \mu \mathrm{l}$ of G1, G2, B1, B2, B3 or B4 to a rubber septum. Bold characters indicate the compounds modified. DMNT indicates $(3 E)$-4,8-dimethyl-1,3,7-nonatriene

\begin{tabular}{|c|c|c|c|c|c|c|c|c|}
\hline \multirow[t]{2}{*}{ Chemicals } & \multicolumn{8}{|c|}{ Synthetic blends (\%) } \\
\hline & $\mathrm{Gc}$ & G1 & $\mathrm{G} 2$ & $\mathrm{Bc}$ & B1 & B2 & B3 & B4 \\
\hline 3-methylbutan-1-ol & 5 & 5 & & 0.6 & 0.6 & 0.6 & 0.6 & 0.6 \\
\hline Butyl acetate & & & & 50 & 50 & & & 50 \\
\hline Pentyl acetate & & & & 3.5 & 3.5 & 3.5 & 3.5 & 3.5 \\
\hline Butyl butanoate & 19.5 & 19.5 & 19.5 & 9 & 9 & 9 & 9 & 9 \\
\hline Propyl hexanoate & 1.5 & & 1.5 & & & & & \\
\hline Butyl hexanoate & 24 & 24 & 24 & 16.8 & 16.8 & 16.8 & 16.8 & 16.8 \\
\hline Hexyl butanoate & 24 & 24 & 24 & 16.8 & 16.8 & 16.8 & 16.8 & 16.8 \\
\hline Pentyl hexanoate & 2.5 & 2.5 & 2.5 & & & & & \\
\hline Pentyl butanoate & 2.5 & 2.5 & 2.5 & 3 & 3 & 3 & 3 & 3 \\
\hline DMNT & 20.5 & 20.5 & 20.5 & & & & 20.5 & 20.5 \\
\hline 1-octen-3-ol & 0.5 & & 0.5 & 0.3 & & & & \\
\hline Total \% & 100 & 98 & 95 & 100 & 99.7 & 49.7 & 70.2 & 120.2 \\
\hline Concentration $(\mu \mathrm{g} / \mu \mathrm{l})$ & 1.0 & 0.98 & 0.95 & 1.0 & 0.997 & 0.497 & 0.702 & 1.202 \\
\hline
\end{tabular}

EAD and GC-MS. Figure $2 \mathrm{a}$ and $\mathrm{b}$ shows GC-EAD recordings from the antennae of a representative green or blueberry hawthorn-origin fly exposed to an extract sample from ripe fruit. We note that whereas the trace indicates the presence of some unidentified peaks with EAD responses, these responses were not present in the majority $(>75 \%)$ of GC-EAD runs examined. The relative ratio of the EAD active compounds estimated by GC-MS for the whole green and blueberry hawthorn fruit adsorbent extracts is given in Table 3.

For green hawthorn (C. viridis), fruit collected from two sites (Dewey Wills, LA, USA 2008; Sharkey Co., MS, USA 2008) were analyzed. A total of 24 different antennal pairs of 21 female and three male green hawthorn-origin flies (1-10 replicate runs/pair) was tested. The compounds that consistently elicited EAD responses were identified as (Ia) 3-methylbutan-1-ol, (IIa) 1-octen-3-ol, (IIIa) butyl butanoate, (IVa) pentyl butanoate, (Va) propyl hexanoate, (VIa) (3E)-4,8-dimethyl-1,3,7-nonatriene (DMNT), (VIIa) butyl hexanoate, (VIIIa) hexyl butanoate, and (IXa) pentyl hexanoate (Table 3).

For Blueberry hawthorn ( $C$. brachyacantha), fruit collected at Ouachita Parish, LA, USA, in 2007 was analyzed. A total of seven different antennal pairs of 5 female and 2 male blueberry hawthorn-origin flies (1-6 replicate runs/pair) was tested using SPME and the extract in the GC-EAD analysis of $C$. brachyacantha volatiles. The corresponding compounds eliciting consistent EAD activity were identified as (Ib) 3-methylbutan-1-ol, (IIb) butyl acetate, (IIIb) 1-octen-3-ol, (IVb) butyl butanoate, (Vb) pentyl butanoate, (VIb) butyl hexanoate, (VIIb) hexyl butanoate, and (VIIIb) pentyl hexanoate (Table 3).

Behavioral Responses of Green and Blueberry HawthornOrigin Flies to Natal Adsorbent Extracts and Non-Natal Northern Volatile Blends A total of 26 green hawthorn-origin flies from Fort Necessity, LA, USA $(N=5)$, Brazos Bend, TX, USA $(N=4)$, and Rolling Fork, MS, USA $(N=17)$ and 17 blueberry hawthorn flies from the Stephen Austin Experimental Forest, near Nacogdoches, TX, USA were tested in the flight tunnel to adsorbent extracts from green and blueberry hawthorn, and to $200 \mu \mathrm{g}$ of the previously developed apple, downy hawthorn, and flowering dogwood blends (Fig. 3). For green hawthorn-origin flies, the response patterns were not statistically different among the three sites for any of the host volatiles tested, and so we present the combined results. A higher proportion of green hawthornorigin flies displayed upwind directed flight to the green hawthorn extract $(69 \%)$ than to the blueberry hawthorn extract $(34 \% ; P=0.002,1 d f)$, the apple blend $(4 \% ; P=0.005$, $1 d f)$, and the downy hawthorn blend $(23 \% ; P=0.002,1 d f)$ (Fig. 3a). No green hawthorn-origin fly responded to the dogwood blend. Similarly, a higher proportion of blueberry hawthorn-origin flies displayed upwind directed flight to the blueberry hawthorn extract $(76 \%)$ than the green hawthorn extract $(24 \% ; P=0.0121,1 d f)$, the downy hawthorn blend 
Fig. 2 Simultaneous recorded GC-EAD responses of adult green and blueberry hawthorn fly antennae to adsorbent samples of green and blueberry hawthorn, respectively. Different traces indicate antennal response of (a) green hawthorn-origin flies to green hawthorn fruit adsorbent samples and (b) blueberry hawthorn-origin flies to blueberry hawthorn fruit adsorbent samples. DMNT indicates $(3 E)-4,8-$ dimethyl-1,3,7-nonatriene

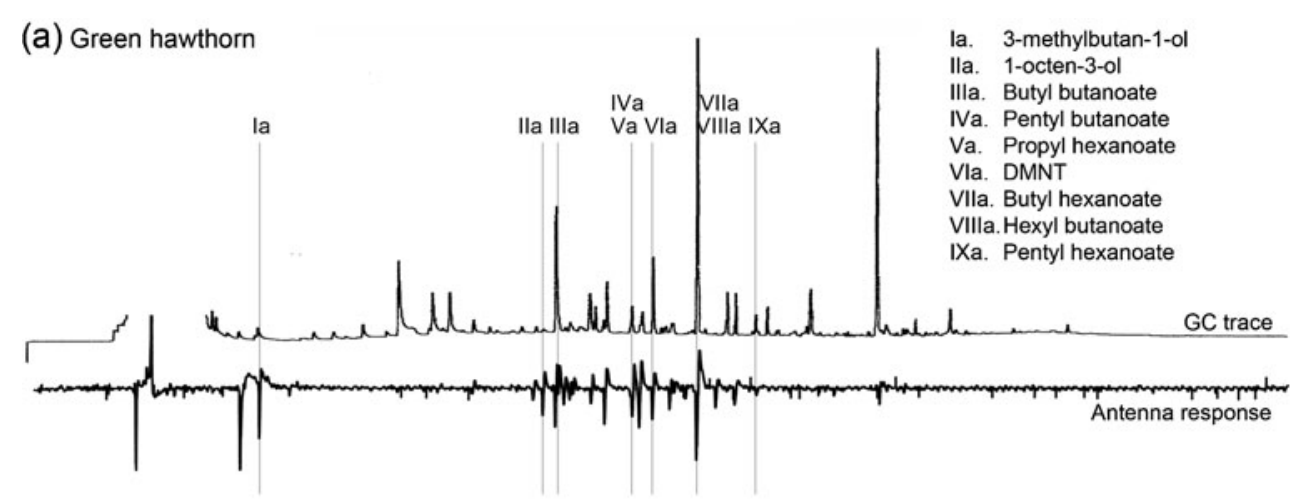

(b) Blueberry hawthorn

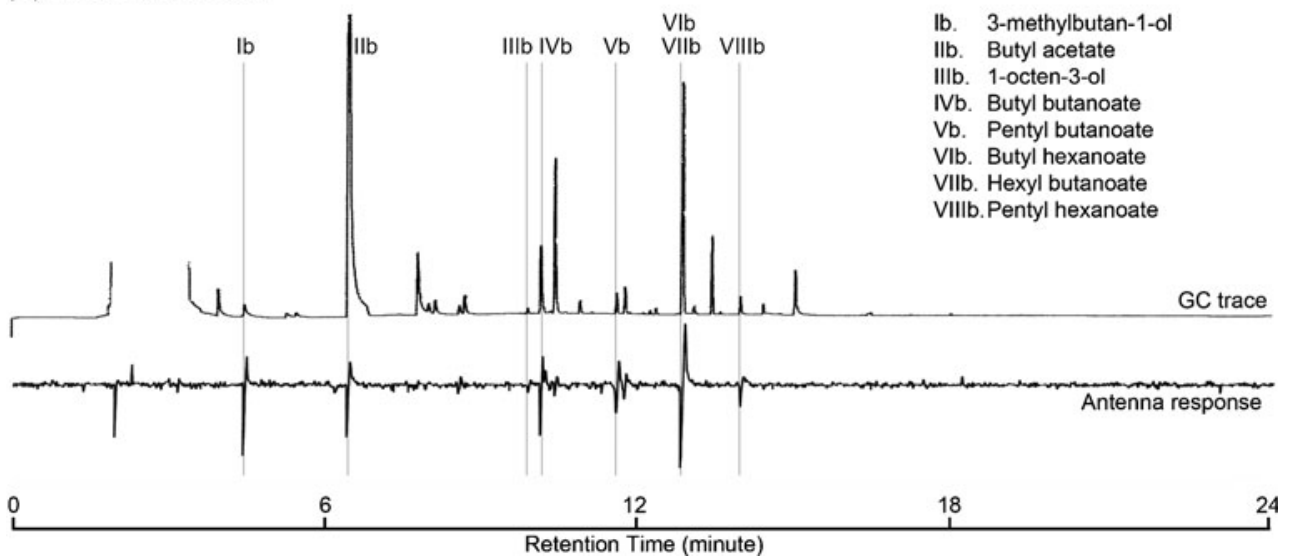

Table 3 The relative ratios (\%) of volatile compounds used in different synthetic blends. Ratios of the green and blueberry hawthorn blends were based on GC/MS analyses of volatile adsorbent samples collected from green and blueberry hawthorn fruits, respectively. The ratio of apple, red hawthorn and dogwood blends were previously developed by Zhang et al. (1999) and Nojima et al. (2003a,b). DMNT indicates (3E)-4,8-dimethyl-1,3,7-nonatriene.

\begin{tabular}{|c|c|c|c|c|c|}
\hline \multirow[t]{2}{*}{ Chemicals } & \multicolumn{5}{|l|}{ Synthetic blends (\%) } \\
\hline & Green hawthorn (Gc) & Blueberry hawthorn (Bc) & Apple & Downy hawthorn & Flowering dogwood \\
\hline 3-methylbutan-1-ol & 5 & 0.6 & & 4 & 27.5 \\
\hline Butyl acetate & & 50 & & & \\
\hline Pentyl acetate & & 3.5 & & & \\
\hline Butyl butanoate & 19.5 & 9 & 10 & & \\
\hline Propyl hexanoate & 1.5 & & 4 & & \\
\hline Butyl hexanoate & 24 & 16.8 & 37 & 0.01 & \\
\hline Hexyl butanoate & 24 & 16.8 & 44 & & \\
\hline Pentyl hexanoate & 2.5 & & 5 & & \\
\hline 3-methylbutyl acetate & & & & 1.5 & 0.9 \\
\hline Pentyl butanoate & 2.5 & 3 & & & \\
\hline Ethyl acetate & & & & 94.32 & 54.9 \\
\hline DMNT & 20.5 & & & 0.07 & \\
\hline Dihydro- $\beta$-ionone & & & & 0.1 & \\
\hline Dimethyl trisulfide & & & & & 1.9 \\
\hline 1-octen-3-ol & 0.5 & 0.3 & & & 9.1 \\
\hline$\beta$-caryophyllene & & & & & 5.8 \\
\hline
\end{tabular}



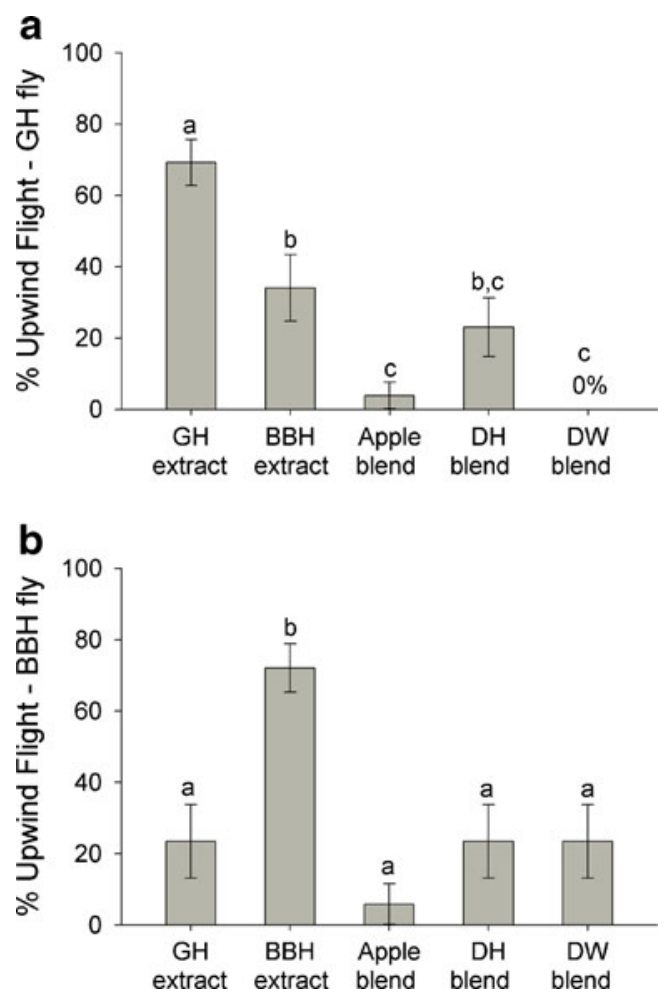

Fig. 3 Upwind flight responses $(\% \pm 1 S E)$ of (a) green hawthornorigin flies and (b) blueberry hawthorn-origin flies to green and blueberry hawthorn fruit adsorbent extracts as well as previously described synthetic volatile blends for three northern USA host plants: apple, downy hawthorn, and flowering dogwood. List of abbreviations used and what they stand for: $\mathrm{GH}$, green hawthorn; $\mathrm{BBH}$, blueberry hawthorn; DH, downy hawthorn; DW, flowering dogwood. Different letters on bars indicate significant differences $(P<0.05)$

(24\%; $P=0.0121,1 d f)$, and the flowering dogwood blend $(24 \% ; P=0.0121,1 d f)$. Only one of the 17 blueberry hawthorn-origin flies $(6 \%)$ responded to the apple blend (Fig. 3b).

Behavioral Response of Green and Blueberry HawthornOrigin Flies to the Synthetic Blends A total of 26 green hawthorn-origin flies from four different sites (Fort Necessity, LA, USA [ $N=8]$; Rolling Fork, MS, USA [ $N=13]$; Brazos Bend, TX, USA [ $N=1]$; and Cedar Lane, TX, USA $[N=4])$ were tested to both the green hawthorn extract and the 9-component synthetic blend developed for green hawthorn fruit (designated Gc). A total of 26 blueberry hawthorn-origin flies from two different sites (Nacogdoches, TX, USA [ $N=19]$; and Marion Ferry, TX, USA $[N=7])$ were tested to the blueberry hawthorn extract and to an 8-component synthetic blend of the complete set of EAD active volatiles identified from fruit (designated $\mathrm{Bc}$ ). For both fly host populations, there was no difference in the level of upwind directed flight with the $\mathrm{Gc}$ or $\mathrm{Bc}$ blend compared to the respective extracts (for green hawthorn 81 vs. $69 \%$, respectively, $P=0.523,1 \mathrm{df}$; for blueberry 88 vs. $65 \%$, respectively, $P=0.098,1 d f)$. These results support the hypothesis that the $\mathrm{Gc}$ and $\mathrm{Bc}$ blends contain all of the key volatiles required for maximal levels of green and blueberry hawthorn upwind flight behavior. We, therefore, used the $\mathrm{Gc}$ and $\mathrm{Bc}$ blends as a baseline for comparison in subsequent tests to investigate the behavioral responses of flies to specific volatile compounds (see section below on Behavioral Responses of Flies to Modified Blends).

Behavioral Responses of Flies to Modified Blends Analysis of the flight responses of green and blueberry hawthornorigin flies to two complete synthetic $(\mathrm{Gc}$ and $\mathrm{Bc})$ and six modified blends (G1, G2, B1, B2, B3, and B4; Table 2) provided evidence for DMNT as a behavioral agonist for green hawthorn flies and a behavioral antagonist for blueberry hawthorn flies, and butyl acetate as a behavioral agonist to blueberry hawthorn flies and a behavioral antagonist for green hawthorn flies (Figs. 4 and 5). There also was evidence for 3-methylbutan-1-ol as an important volatile for maximal upwind flight levels by both green and blueberry hawthorn flies, while 1-octen-3-ol (a critical volatile for the dogwood flies; Linn et al., 2005b) and propyl hexanoate (found in the apple blend) had little influence on fly behavior.

For the 85 green hawthorn flies from Dewey Wills, LA, USA $(N=20)$; Fort Necessity, LA, USA $(N=26)$; Rolling Fork, MS, USA $(N=28)$; Brazos Bend, TX, USA $(N=4)$; and Cedar Lane, TX, USA $(N=7)$, there was no significant difference in the level of upwind directed flight between the complete Gc blend and the G1 blend (Gc minus 1-octen-3-ol and propyl hexanoate) (73 vs. $78 \% ; P=0.594,1 d f)$, suggesting that 1-octen-3-ol and propyl hexanoate were not essential for maximal upwind flight responses by green hawthorn flies (Fig. 4a). Of the 85 flies, 40 (Dewey Wills, LA, USA [ $N=20]$; Fort Necessity, LA, USA [ $N=11]$; Rolling Fork, MS, USA [ $N=6]$; and Brazos Bend, TX, USA [ $N=3]$ ) also were tested against blend G2 (Gc minus 3-methylbutan1-ol), with upwind directed flight responses significantly lower than with Gc (10 vs. $78 \% ; P<0.001,1 d f)$. In addition, the same 26 green hawthorn flies tested above to both the green hawthorn adsorbent extract and $\mathrm{Gc}$ were tested to the complete blueberry hawthorn blend $(\mathrm{Bc})$ and modified $\mathrm{B} 1$ (Bc minus 1-octen-3-ol). Green hawthorn flies exhibited low response levels to both Bc and B1 (23 and 4\% upwind flight behavior; Fig. 4b).

To test the effect of butyl acetate and DMNT on green hawthorn-origin flies, we performed the following comparisons. The 85 green hawthorn flies tested above to $\mathrm{Gc}$ and G1 also were tested to modified blueberry hawthorn blend B2 (B1 minus butyl acetate), to which they showed an increased level of upwind directed flight compared with $\mathrm{Bc}$ (47 vs. $24 \% ; P=0.04$; Fig. $4 \mathrm{~b}$ ), suggesting that butyl 

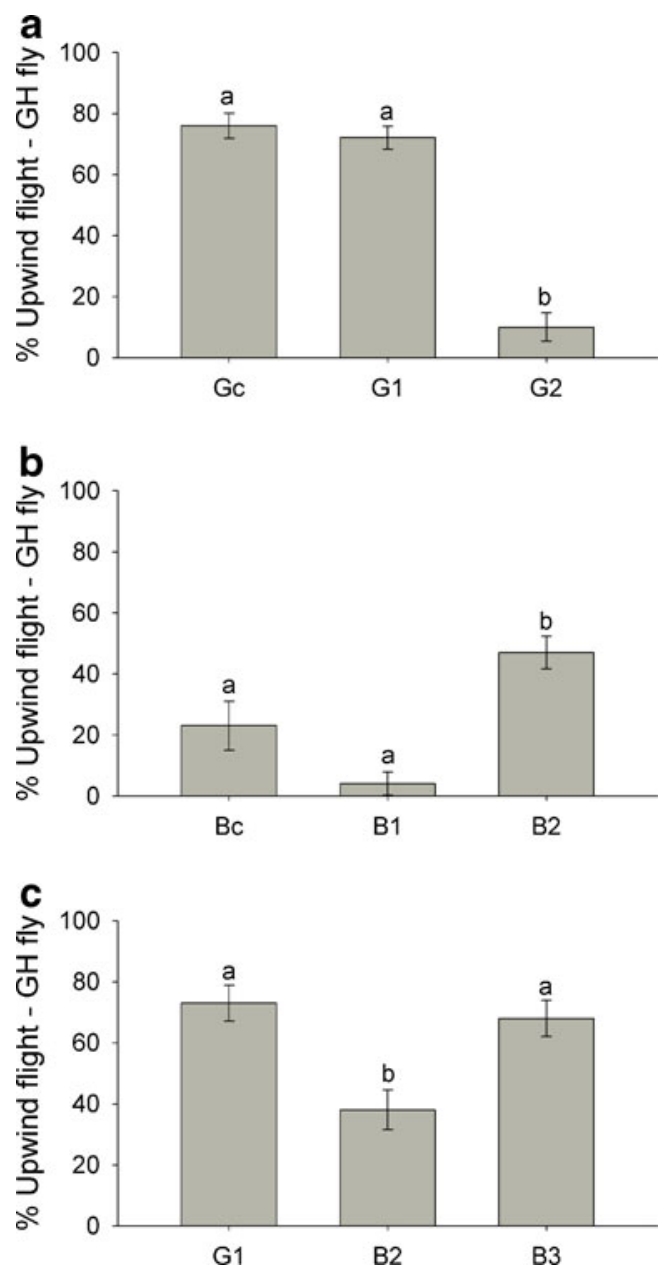

Fig. 4 Upwind flight responses $(\% \pm 1 S E)$ of green hawthornorigin flies to (a) Gc: the complete green hawthorn synthetic blend, G1: a modified synthetic green hawthorn blend missing 1-octen-3-ol and propyl hexanoate, and G2: a modified version of Gc missing 3methylbutan-1-ol, (b) Bc: the complete blueberry hawthorn synthetic blend, B1: a modified blueberry hawthorn blend missing 1-octen-3ol, and B2: a version of B1 missing butyl acetate, and (c) G1, B2, and B3: a modified version of B2 containing DMNT. GH indicates green hawthorn. Different letters on bars indicate significant differences $(P<0.05)$

acetate, a unique compound to blueberry hawthorn fruit among the southern hawthorn species, functions as an interspecific behavioral antagonist on the flight behavior of green hawthorn flies. A total of 56 additional green hawthorn flies from Brazos Bend, TX, USA $(N=31)$, Dewey Wills, LA, USA $(N=18)$, Marion Ferry, TX, USA $(N=5)$, and Palmetto State Park, TX, USA $(N=2)$, were tested to the modified blueberry blend B3, as well as to B2 and G1 (Fig. 3c). Modified blend B3 represented blend B2 with the compound DMNT added, making B3 very similar in composition (except for pentyl acetate in B3) to the modified G1 blend. For these 56 green hawthorn flies, the upwind directed flight response to B3 $(68 \%)$ was higher compared to $\mathrm{B} 2(38 \% ; P<0.001,1 d f)$ and was similar to
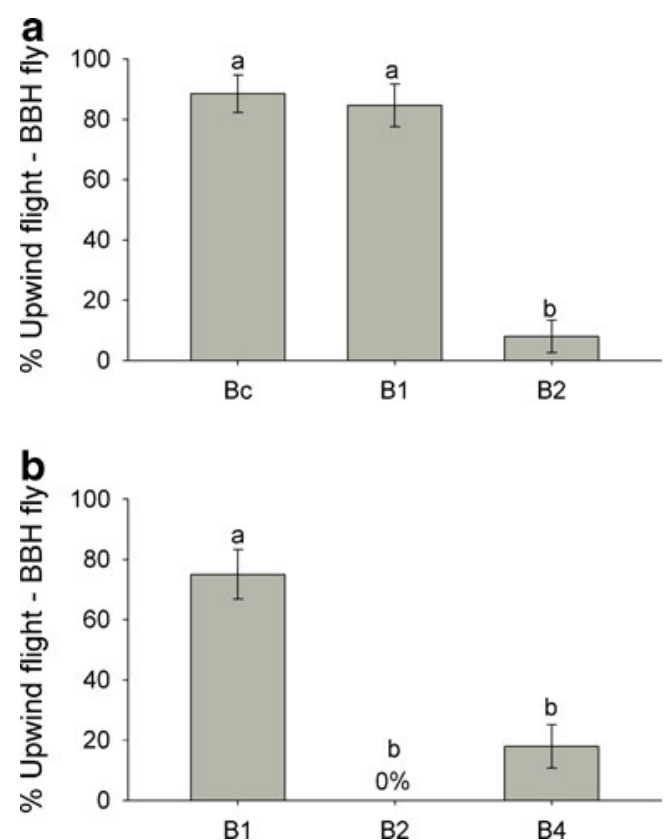

Fig. 5 Upwind flight responses $(\% \pm 1 S E)$ of blueberry hawthornorigin flies to (a) Bc: the complete blueberry hawthorn synthetic blend, B1: a modified version of Bc missing 1-octen-3-ol, and B2: a modified version of B1 missing butyl acetate, and (b) B1, B2, and B4: a modified version of B1 containing DMNT. BBH indicates blueberry hawthorn. Different letters on bars indicate significant differences $(P<0.05)$

that for G1 $(73 \% ; P=0.66)$, suggesting that DMNT is a critical volatile in the blend that green hawthorn flies use to locate green hawthorn trees (Fig. 4c).

To test the effect of 1-octen-3-ol, butyl acetate, and DMNT on blueberry hawthorn flies, we performed the following comparisons. For the 26 blueberry hawthornorigin flies tested above from Nacogdoches, TX, USA $(N=19)$, and Marion Ferry, TX, USA $(N=7)$, there was no significant difference in the level of upwind directed flight between the complete $\mathrm{Bc}$ blend and the modified $\mathrm{B} 1$ blend (Bc minus 1-octen-3-ol) (88 and 85\% upwind flight, respectively), suggesting that 1-octen-3-ol does not greatly affect upwind flight by blueberry hawthorn flies (Fig. 5a). However when butyl acetate was removed from the B1 blend to create blend B2, fewer blueberry hawthorn flies exhibited upwind directed flights (8\%) compared with B1 $(88 \% ; P<0.001,1 d f)$, supporting the essential role of butyl acetate for blueberry hawthorn flies (Fig. 5a). Further support for a major role of butyl acetate came from a test of 28 blueberry flies collected from Lufkin, TX, USA in 2009. These flies also displayed a dramatic decrease in response when butyl acetate was not present in the blueberry hawthorn blend (75\% upwind flight to $\mathrm{B} 1$ vs. $0 \%$ to $\mathrm{B} 2 ; P<0.001,1 d f$ ) (Fig. 5b). Moreover, addition of DMNT to B1 to create modified blend B4 also reduced upwind directed flight behavior for the 28 Lufkin, TX, USA, flies $75 \%$ upwind 
flight to $\mathrm{B} 1$ vs. $18 \%$ to $\mathrm{B} 4 ; P<0.001,1 d f$ ), suggesting that blueberry hawthorn-origin fly flight behavior was antagonized by the presence of DMNT, a key green hawthorn volatile (Fig. 5b) in the mixture.

\section{Discussion}

We developed synthetic volatile blends for green (C. viridis) and blueberry hawthorn (C. brachyacantha) fruit using GCEAD and GC-MS analysis of SPME and adsorbent samples. In the flight tunnel, green and blueberry hawthorn-origin flies displayed maximal levels of upwind directed flight to their respective natal blends and reduced response levels to their non-natal blends. The reduced response levels were due to differences in key volatiles that comprised the behaviorally active blend for each host, and to the presence of non-natal fruit volatiles that function as behavioral antagonists.

Two key differences were discovered between the green and blueberry hawthorn blends. The first was the presence of DMNT in the green hawthorn blend and its absence from the blueberry hawthorn blend. Addition and subtraction of DMNT to synthetic green and blueberry hawthorn blends demonstrated the critical importance of the compound for stimulating upwind oriented flight for green hawthorn-origin flies and for antagonizing the upwind flight of blueberry hawthorn flies. The compound DMNT has not been found in the fruit of other southern hawthorn species besides green hawthorn (blueberry hawthorn and three mayhaw species; Cha et al., 2011b, this issue), suggesting that it may be a unique volatile in the blend distinguishing $C$. viridis. The second difference is the presence of butyl acetate in the blueberry hawthorn blend and its absence from the green hawthorn blend. Addition and subtraction of butyl acetate to the respective synthetic blends showed the critical importance of this volatile for stimulating upwind oriented flight in blueberry flies and for antagonizing the upwind flight of green hawthorn flies. The striking contrast in preference behavior indicates that southern $R$. pomonella flies that infest green vs. blueberry hawthorn differ in their host odor response phenotypes. Butyl acetate has not been found in the fruit of other southern hawthorn species that co-occur with the blueberry hawthorn across its range in eastern Texas and Louisiana (e.g., western mayhaw, C. opaca), although the compound is present in the more eastern distributed mayhaws, $C$. aestivalis and C. rufula (Cha et al., 2011b, this issue). Butyl acetate may, therefore, be a key compound in the blend that distinguishes $C$. brachyacantha throughout its geographic distribution in the South. The results for DMNT and butyl acetate continue a pattern we first observed in northern $R$. pomonella populations (Linn et al., 2005a, b) involving positive orientation of flies to key mixtures of volatile components present in the adsorbent extract profiles of their natal host fruit, and antagonism or arrestment of upwind flight when unique compounds found in alternative non-natal fruit are added to the natal blend. Antagonism is important because it indicates that when flies shift to a new host plant, not only do they evolve to positively behaviorally respond to the volatile profile of the new fruit (where offspring survivorship is presumably higher) but also to avoid certain volatiles of other, alternative host fruit (where fitness should be lower).

The green hawthorn and blueberry hawthorn synthetic blends also showed similarities. Perhaps noteworthy, both blends, as well as the northern downy hawthorn, the dogwood blend, and the three southern mayhaw blends, share 3-methybutan-1-ol in common. Moreover, 3methybutan-1-ol is not present in the apple blend, and is a potent behavioral antagonist for apple-origin fly flight behavior (Linn et al., 2005a). Our current results, therefore, further underscore that 3-methybutan-1-ol may be an essential component of behaviorally active volatile blends for hawthorn infesting $R$. pomonella flies. In comparison, the volatile 1-octen-3-ol that we previously showed to be a key agonist volatile for dogwood fly behavior and a behavioral antagonist for apple flies (Linn et al., 2005a), while present in both the green hawthorn and blueberry hawthorn blend, did not significantly affect the upwind flight of green or blueberry hawthorn flies.

Because of limitations in the number of available flies, we were not able to conduct exhaustive subtraction assays for all of the compounds in the respective natal blends. Thus, it is not possible to definitively say whether any of the remaining compounds in the green hawthorn and blueberry hawthorn synthetic blends (e.g., butyl hexanoate and hexyl butanoate) are essential for behavioral activity and discrimination. We also recognize that important differences in the ratio of common compounds could be important (e.g., Cha et al., 2011a). For example, butyl hexanoate is an important volatile for the northern downy hawthorn (C. mollis) flies, but is present in very low quantities compared to the apple blend $(<1$ vs. 44\%; Nojima et al., 2003a), and high proportions of butyl hexanoate in the northern downy hawthorn blend have been shown to act antagonistically on upwind flight responses (Linn et al., 2005a).

Coupled with our results for three mayhaw host plants in the southern USA (Cha et al., 2011b, this issue), the current study does not support the hypothesis that the introduction of a geographically differentiated, southern hawthorninfesting form of $R$. pomonella was the direct source of the apple-infesting race of the fly in the North (Carson, 
1989; see Bush et al., 1989). In the current study, flies collected from both green and blueberry hawthorn fruit exhibited low response levels with the apple blend in flight tunnel assays ( 0 and $6 \%$ upwind directed flight, respectively). Indeed, the proportion of upwind directed flight exhibited by southern green hawthorn and blueberry hawthorn-origin flies to the apple blend was lower than that shown for the downy hawthorn blend (27 and 25\%, respectively). Green and blueberry hawthorn-infesting populations of $R$. pomonella therefore do not preferentially orient to apple and are not "pre-adapted", direct precursors to the apple race in the North. However, just like southern mayhaws (Cha et al., 2011b, this issue), both green and blueberry hawthorn fruit from the South also contain certain compounds present in the apple blend and absent in the fruit of the downy hawthorn, the major hawthorn host attacked by $R$. pomonella in the North. In particular, mayhaw, green hawthorn, and the apple blend all contain butyl butanoate, propyl hexanoate, butyl hexanoate, hexyl butanoate, and pentyl hexanoate. Blueberry and green hawthorn flies are thus capable of detecting certain components of the apple blend, and some southern flies are capable of responding to both hawthorn and apple blends, supporting the hypothesis that odor response phenotypes in the South may have contributed to prestanding variation in northern hawthorn populations facilitating a rapid, sympatric shift to apple. Rather than a preadapted apple race in the South, our results instead provide more evidence for the possible existence of differentiated races (or even sibling species) of hawthorn-infesting $R$. pomonella in the South.

We now have developed active synthetic blends for eight different host fruit of $R$. pomonella group flies. With the addition of the green hawthorn and blueberry hawthorn blends in the current study, we have now characterized fruit volatile profiles for most of the major hawthorn hosts infested by $R$. pomonella across the northern and southern USA. Development of these blends will allow us to further characterize phenotypic patterns and elucidate the genetic architecture of host plant preference in the classic $R$. pomonella system, leading to a greater understanding of the role that host volatile recognition plays in phytophagous insect speciation (Smadja and Butlin, 2009). The importance of pre-standing variation in hawthorn flies to recognize certain specific fruit volatiles in apple may now become clearer as we approach a complete picture of host race formation among southern $R$. pomonella populations.

Acknowledgments We thank Tracy Arcella, Stewart Berlocher, Scot Egan, Andrew Forbes, Glen Hood, Andy Michel, Matt Michel, Jim Stevens, Sheina Sim, and David Riskind of Texas State Parks for help in collecting fruit and fly samples, thoughtful discussion, and/or assistance in helping us prepare the manuscript for publication. We also thank Callie Musto, Kathy Poole, and Paula Fox for maintaining the flies received from Notre Dame, and Harvey Reissig, Dave Combs, and Cynthia Smith for use of the Geneva, NY apple maggot colony. We also thank Aijun Zhang for the synthesis of $(3 E)-4,8$ dimethyl-1,3,7-nonatriene. The research was supported by grants to J. L.F. and C.L. from the National Science Foundation and to J.L.F. from the USDA.

\section{References}

BERLOCHER, S. H., and FEDER, J. L. 2002. Sympatric speciation in phytophagous insects: moving beyond controversy? Annu. Rev. Entomol. 47: 773-815.

BERLOCHER, S. H., MCPHERON, B. A., FEDER, J. L., and BUSH, G. L. 1993. A revised phylogeny of the Rhagoletis pomonella (Diptera: Tephritidae) sibling species group. Ann. Ent. Soc. Amer. 86:716-727.

BUSH, G. L., FEDER, J. L., BERLOCHER, S. H., MCPHERON, B. A., SMITH, D. C., and CHILCOTE, C. A. 1989. Sympatric origins of $R$. pomonella. Nature 339: 346.

CARSON, H. L. 1989. Sympatric pest. Nature 338: 304.

CHA, D. H., LINN, C. E., TEAL, P. E. A., ZHANG, A., ROELOFS, W. L., and LOEB, G. M. 2011a. Eavesdropping on plant volatiles by a specialist moth: significance of ratio and concentration. PLOS ONE 6:e17033.

CHA, D. H., POWELL, H. Q., FEDER, F. L., and LINN, C. E. JR. 2011b. Identification of host fruit volatiles from Mayhaw (Crataegus series Aestivalis) attractive to Mayhaw-origin Rhagoletis pomonella flies. J. Chem. Ecol. 37: this issue.

CORRELL, D. S., and JOHNSTON, M. C. 1970. Manual of the vascular plants of Texas. Vol. 6 Texas Research Foundation, $1881 \mathrm{p}$.

DAMBROSKI, H. R., and FEDER, J. L. 2006. Host plant and latitude-related diapause variation in Rhagoletis pomonella: a test for multifaceted life history adaptation on different stages of diapauses development. J. Evol. Biol. 20: 21012112 .

FEDER, J. L. 1998. The apple maggot fly, Rhagoletis pomonella: flies in the face of conventional wisdom? pp. 130-144, in D. J. Howard and S. H. Berlocher, (eds.). Endless Forms: Species and Speciation. Oxford University Press, Oxford.

FEIN, B. L., RESSIG, W. H., and ROELOFS, W. L. 1982. Identification of apple volatiles attractive to the apple maggot. J. Chem. Ecol. 8: 1473-1487.

FUNK, D. J., FILCHAK, K. E., and FEDER, J. L. 2002. Herbivorous insects: model systems for the comparative study of speciation ecology. Genetica 116: 251-267.

GREENWALD, R., CHAYKOVSKY, M., and COREY, E. J. 1963. The Wittig reaction using methylsulfinyl carbanion-dimethyl sulfoxide. J Org. Chem. 28:1128-1129.

LINN, C. E., NOJIMA, S., and ROELOFS, W. L. 2005a. Antagonist effects of non-host fruit volatiles on discrimination of host fruit by Rhagoletis flies infesting apple (Malus pumila), hawthorn (Crataegus spp.), and flowering dogwood (Cornus florida). Entomol. Exp. Appl. 114: 97-105.

LINN, C. E., DAMBROSKI, H., NOJIMA, S., FEDER, J. L., BERLOCHER, S. H., and ROELOFS, W. L. 2005b. Variability in response specificity of apple, hawthorn, and flowering dogwood-infesting Rhagoletis flies to host fruit volatile blends: implications for sympatric host shifts. Entomol. Exp. Appl. 116: $55-64$.

NEILSON, W. T. A., and McALLAN, J. W. 1965. Artificial diets for apple maggot .3. improved defined diets J. Econ. Entomol. 58: 542. 
NOJIMA, S., LINN, C. E., JR, ZHANG, A., MORRIS, B., and ROELOFS, W. L. 2003a. Identification of host fruit volatiles from hawthorn (Crataegus spp.) attractive to hawthorn-origin Rhagoletis pomonella flies. J. Chem. Ecol. 29: 319-334.

NOJIMA, S., LINN, C. E., JR, ZHANG, A., Morris, B., and ROELOFS, W. L. 2003b. Identification of host fruit volatiles from flowering dogwood (Cornus florida) attractive to dogwoodorigin Rhagoletis pomonella flies. J. Chem. Ecol. 29: 2347-2357.

SARGENT, C. S. 1890. Silva of North America Volume iv. (reprinted 1947) Peter Smith, New York, NY.

SARGENT, C. S. 1933. Manual of the Trees of North America. Houghton Mifflin Co., Boston, MA.
SMADJA, C., and BUTLIN, R. K. 2009. On the scent of speciation: the chemosensory system and its role in premating isolation. Heredity. 102: 77-97.

TALENT, N., and DICKINSON, T. A. 2005. Polyploidy in Crataegus and Mespilus (Rosaceae, Maloideae): evolutionary inferences from flow cytometry of nuclear DNA amounts. Can. J. Bot. 83: 1268-1304.

VINES, R. A. 1977. Trees of East Texas. University of Texas Press, Austin, TX.

ZHANG, A., LINN, C. E., JR, WRIGHT, S., PROKOPY, R., RESSIG, W., and ROELOFS, W. L. 1999. Identification of a new blend of apple volatiles attractive to the apple maggot, Rhagoletis pomonella. J. Chem. Ecol. 25: 1221-1232. 